\title{
Tendência de AIDS no grupo etário de 50 anos e mais no período anterior e posterior à introdução de medicamentos para disfunção erétil: Brasil, 1990 a 2003
}

\author{
AIDS trend in the group of 50-year-old people and more in the \\ previous and subsequent period to the introduction of \\ medicines for erectile dysfunction: Brazil, 1990 to 2003
}

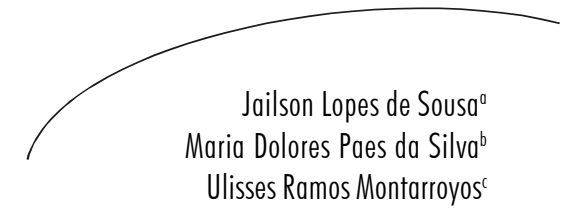

Maria Dolores Paes da Silva

\section{Resumo}

Objetivo: Descrever o comportamento da incidência de Aids no grupo etário de 50 anos e mais, no Brasil e suas macrorregiões, no período de 1990 a 2003, analisando o momento anterior e posterior à introdução dos medicamentos para disfunção erétil no país, em 1998. Procedimentos metodológicos: Realizou-se estudo ecológico, através de análise espaço-temporal dos casos de Aids notificados. Modelos de regressão linear, ajustados à série temporal dos casos de Aids com data de diagnóstico de 1990 a 1997, forneceram as taxas de variação média anual, bem como os valores esperados, possibilitando, dessa forma, a comparação das taxas de incidência observadas e estimadas. Resultados: Foram analisados 25.223 casos de Aids no grupo etário de 50 anos e mais, estando a grande maioria (84\%) situados nas regiões Sudeste e Sul do país. Essas regiões também apresentaram as maiores taxas de variação média anual até o ano de 1997 (1,002 e 0,906, respectivamente), seguidas de Centro-Oeste (0,570), Norte $(0,441)$ e Nordeste $(0,254)$, apresentando o Brasil uma taxa de 0,588 . Após 1998, a comparação das taxas acumuladas no período de 1998 a 2003 revelou tendências esperadas maiores do que as observadas em todas as regiões, exceto a Nordeste. Conclusão: A tendência de crescimento da Aids na faixa etária de 50 anos e mais apresentou maiores taxas de crescimento no período entre $1990 \mathrm{e}$ 1997, mantendo esse crescimento no período posterior à introdução dos medicamentos para disfunção erétil, só que em menor velocidade.
Palavras-chave:

Síndrome da Imunodeficiência Adquirida; envelhecimento; impotência; preparações farmacêuticas; estudos de coortes; meia-idade; Brasil

Correspondência / Correspondência

Jailson Lopes de Sousa

Av. Múcio Uchôa Cavalcanti, 510, bloco B, apt ${ }^{\circ}$ 201, Engenho do Meio

50730-670, Recife, PE, Brasil

E-mail: jailsonsaudecoletiva@yahoo.com.br 


\section{Abstract}

Objective: Describe the behavior of the incidence of Aids in the group of 50year-old people and more, in Brazil and its macro-regions, from 1990 to 2003, analyzing the previous and subsequent moment of introduction of medicines for erectile dysfunction in the country, in 1998. Methods: An ecological study was carried out, along with a space-time analysis of the notified cases of Aids in this group of people. Models of linear regression, adjusted to the time series of Aids cases whose diagnoses date from 1990 to 1997, provided the taxes of annual average variation, as well as the expected values, so making it possible to compare incidence taxes observed and estimated from 1998 to 2003. Results: 25,223 cases of Aids in the group of 50-year-old people and more had been analyzed, being the majority $(84 \%)$ in the Southeast and South regions. Those regions also presented the greatest taxes of annual average variation to the year of 1997 (1.002 and 0.906, respectively), followed by the Middle-West (0.570), North (0.441) and Northeast (0.254); Brazil showed a tax of 0.588 . After 1998, the comparison of the taxes accumulated from 1998 to 2003 revealed the expected higher trends than the ones observed in all regions, except for the Northeast. Conclusion: The trend of growth of Aids in the 50-year-old people and more group presented greater taxes of growth from 1990 to 1997, and this growth remained in the period subsequent to the introduction of medicines for erectile dysfunction, but more slowly.

Key words: Acquired Immunodeficiency Syndrome; aging; impotence; pharmaceutical preparations; cohort studies; middle aged; Brazil

\section{INTRODUÇÃO}

Têm-se percebido mudanças no curso da epidemia de Aids, atualmente, e uma delas é o aumento do número de casos na faixa etária de 50 anos e mais. Apesar disso, a Aids na maturidade mostra que esses cidadãos ainda estão invisíveis no que diz respeito às políticas públicas de prevenção. Embora minoria, os adultos maduros e idosos são mais atingidos, em todo o país, até mesmo do que crianças e adolescentes, considerados prioridade pelos gestores: de 1980 a junho de 2006, 26.014 casos de Aids foram notificados em maiores de 50 anos, contra 10.031 e 5.034 notificações entre crianças e adolescentes, respectivamente. ${ }^{4}$

Muitos investimentos têm sido feitos para melhorar a qualidade de vida dos idosos, quando esta população começa a viver a vida em sua plenitude, com aumento das relações sociais e, conseqüentemente, sexuais, já que a libido não acaba com o aumento da idade. ${ }^{3} \mathrm{Com}$ isso, riscos antes característicos de faixas etárias mais jovens estão cada vez mais acometendo pessoas a partir dos 50 anos. Com a estimativa do aumento expressivo no número de idosos, surge um grande desafio para o Brasil: o estabelecimento de políticas públicas e estratégias que 
possam garantir a qualidade de vida dessas pessoas. Portanto, questões como a Aids no envelhecimento devem ser aprofundadas, de modo a fornecer subsídios tanto para os cuidados com os portadores do HIV e da Aids, como no desenvolvimento de ações e programas de prevenção. ${ }^{1}$

Dessa forma, estudos que se proponham a descrever prováveis fatores relacionados com a incidência do HIV e Aids nesse grupo etário se justificam por vários motivos. Primeiro, pela necessidade de se discutir a difusão dos medicamentos para disfunção erétil a partir de 1998 no Brasil, numa parcela da população cada vez mais crescente, que não teve o hábito de lidar com métodos preventivos em décadas passadas e que não se sente vulnerável às DST. Segundo, no que pesem esses dados, a literatura científica relativa a riscos para o HIV/ Aids esteve predominantemente preocupada com as características de risco em outros grupos da população, como adultos jovens, adolescentes e crianças. E por último, pela possibilidade de direcionamento de campanhas preventivas, focadas para este grupo populacional específico, até então excluído das discussões de vulnerabilidade ao HIV e Aids.

Sendo assim, este estudo teve como objetivo descrever o comportamento da incidência de Aids no grupo etário de 50 anos e mais, no Brasil e suas macrorregiões, no período de 1990 a 2003, analisando o momento anterior e posterior à introdução de medicamentos para disfunção erétil.

\section{PROCEDIMENTOS METODOLÓGICOS}

A área de estudo correspondeu ao Brasil e suas cinco macrorregiões: Norte, Nordeste, Sudeste, Sul e Centro-Oeste. A população estudada foi constituída por todos os casos de Aids em pessoas com idade igual ou superior a 50 anos, notificados ao Sistema de Informação de Agravos de Notificação (SINAN), com ano de diagnóstico de 1990 a 2003. Os dados foram obtidos no endereço eletrônico do DATASUS (http:// www.datasus.gov.br). Para evitar erros de retardo de notificação, optou-se por analisar os casos de Aids até 2003, por se tratar do último ano encerrado no banco de dados do SINAN.

Os dados populacionais do Brasil e Grandes Regiões que subsidiaram a construção dos coeficientes de incidência de Aids foram obtidos na Fundação Instituto Brasileiro de Geografia e Estatística (FIBGE), tomando como base os censos demográficos de 1980,1991 e 2000 , e a contagem populacional de 1996, os quais serviram de referência para a construção das projeções populacionais dos anos inter e pós-censitários pelo método geométrico.

Tratou-se de estudo ecológico, descritivo e de base populacional, sendo realizada análise espaço-temporal dos casos de Aids notificados na população de estudo. Para análise dos dados foram utilizados modelos de regressão linear, ajustados à série temporal da incidência de Aids na faixa etária de 50 anos ou mais, no período anterior à introdução dos medicamentos para disfunção erétil no Brasil (1990 a 1997). 
Esses modelos forneceram as taxas de variação média anual, as quais possibilitaram o cálculo das incidências de Aids esperadas, nessa faixa etária, para o período posterior à introdução dos medicamentos para disfunção erétil (1998 a 2003), possibilitando, dessa forma, a comparação dos valores observados e esperados para o período citado.

O presente projeto de pesquisa foi submetido e aprovado pelo Comitê de Ética em Pesquisa com Seres Humanos da Universidade Federal de Pernambuco, estando de acordo com a Resolução No 196/96, do Conselho Nacional de Saúde.

\section{RESULTADOS}

Foram analisados 25.223 casos de Aids no grupo etário de 50 anos e mais notificados ao SINAN até junho de 2006, com data de diagnóstico entre 1990 e 2003 (Tabela 1). A maioria dos casos ocorreu nas regiões Sudeste e Sul, que alcançaram juntas, em cada ano da série estuda, um percentual acima de $80 \%$ dos casos de todo o país, terminando o acumulado do período com quase $84 \%$ da totalidade dos casos. É ainda importante salientar a baixa participação das regiões Nordeste, Centro-Oeste e Norte nos casos de Aids notificados, sendo responsáveis por apenas $16,41 \%$ dos casos acumulados em todo o período.

Tabela 1 - Número e percentual de casos de Aids notificados no grupo etário de 50 anos e mais segundo ano de diagnóstico e macrorregiões, Brasil, 1990-2003

\begin{tabular}{|c|c|c|c|c|c|c|c|c|c|c|c|c|}
\hline \multirow[b]{2}{*}{ Ano } & \multicolumn{2}{|c|}{$\mathrm{N}$} & \multicolumn{2}{|c|}{$\mathrm{NE}$} & \multicolumn{2}{|c|}{$\mathrm{SE}$} & \multicolumn{2}{|c|}{$\mathrm{S}$} & \multicolumn{2}{|c|}{$\mathrm{CO}$} & \multicolumn{2}{|c|}{ BRASIL } \\
\hline & $\mathrm{N}$ & $\%$ & $\mathrm{~N}$ & $\%$ & $\mathrm{~N}$ & $\%$ & $\mathrm{~N}$ & $\%$ & $\mathrm{~N}$ & $\%$ & $\mathrm{~N}$ & $\%$ \\
\hline 1990 & 1 & 0,16 & 54 & 8,84 & 468 & 76,60 & 65 & 10,64 & 23 & 3,76 & 611 & 100 \\
\hline 1991 & 7 & 0,92 & 50 & 6,61 & 579 & 76,49 & 89 & 11,76 & 32 & 4,23 & 757 & 100 \\
\hline 1992 & 10 & 1,12 & 70 & 7,81 & 717 & 80,02 & 71 & 7,92 & 28 & 3,13 & 896 & 100 \\
\hline 1993 & 13 & 1,18 & 92 & 8,36 & 811 & 73,66 & 124 & 11,26 & 61 & 5,54 & 1101 & 100 \\
\hline 1994 & 9 & 0,75 & 104 & 8,67 & 840 & 70,06 & 192 & 16,01 & 54 & 4,50 & 1199 & 100 \\
\hline 1995 & 15 & 1,02 & 137 & 9,29 & 1022 & 69,34 & 229 & 15,54 & 71 & 4,82 & 1474 & 100 \\
\hline 1996 & 35 & 2,15 & 173 & 10,61 & 1083 & 66,40 & 258 & 15,82 & 82 & 5,03 & 1631 & 100 \\
\hline 1997 & 29 & 1,54 & 166 & 8,83 & 1289 & 68,53 & 308 & 16,37 & 89 & 4,73 & 1881 & 100 \\
\hline 1998 & 39 & 1,77 & 209 & 9,48 & 1430 & 64,85 & 436 & 19,77 & 91 & 4,13 & 2205 & 100 \\
\hline 1999 & 45 & 2,07 & 231 & 10,61 & 1395 & 64,05 & 415 & 19,05 & 92 & 4,22 & 2178 & 100 \\
\hline 2000 & 47 & 2,05 & 244 & 10,62 & 1422 & 61,88 & 489 & 21,28 & 96 & 4,18 & 2298 & 100 \\
\hline 2001 & 37 & 1,58 & 245 & 10,44 & 1452 & 61,89 & 499 & 21,27 & 113 & 4,82 & 2346 & 100 \\
\hline 2002 & 103 & 3,34 & 309 & 10,03 & 1847 & 59,97 & 647 & 21,01 & 174 & 5,65 & 3080 & 100 \\
\hline 2003 & 112 & 3,14 & 325 & 9,11 & 2173 & 60,94 & 734 & 20,58 & 222 & 6,23 & 3566 & 100 \\
\hline TOTAL & 502 & 1,99 & 2409 & 9,55 & 16528 & 65,53 & 4556 & 18,06 & 1228 & 4,87 & 25223 & 100 \\
\hline
\end{tabular}

Fonte: SINAN, Ministério da Saúde. 
O país apresentou uma incidência de Aids em pessoas com 50 anos e mais, no período de 1990 a 2003, de 7,45 casos por 100.000 habitantes. Em relação às macrorregiões, a incidência total também é maior nas regiões Sudeste (10,5/100.000) e Sul (8,3/100.000), seguidas pelas regiões Centro-Oeste $(7,0 /$ 100.000), Norte $(3,0 / 100.000)$ e Nordeste $(2,65 / 100.000)$.

As taxas de variação média anual para o período anterior à introdução de medicamen- tos para disfunção erétil no país (1990 a 1997) revelam uma tendência de crescimento anual da incidência de Aids na faixa etária estudada estatisticamente significativa ( $\mathrm{p}<0,001)$, tanto para o Brasil $(0,588)$ como para todas as macrorregiões (Tabela 2). Destacam-se as regiões Sudeste e Sul, com as maiores taxas de crescimento médio, de 1,002 e 0,906, unidade de incidência de Aids por 100.000 habitantes ao ano, respectivamente, seguidas das regiões Centro-Oeste $(0,570)$, Norte $(0,441)$ e Nordeste $(0,254)$.

Tabela 2 - Componentes da equação da reta, p valor e coeficiente de determinação, segundo macrorregiões, Brasil, 1990-1997

\begin{tabular}{lcccc}
\hline Macrorregião & $\begin{array}{c}\text { Taxa de variação } \\
\text { média anual }(\beta)\end{array}$ & Constante $(\alpha)$ & Valor de $\mathrm{p}$ & $\begin{array}{c}\text { Coeficiente de } \\
\text { Determinação }\left(\mathrm{R}^{2}\right)\end{array}$ \\
\hline Norte & 0,441 & $-877,768$ & $\mathrm{p}<0,001$ & 0,785 \\
Nordeste & 0,254 & $-505,105$ & $\mathrm{p}<0,001$ & 0,968 \\
Sudeste & 1,002 & $-1992,769$ & $\mathrm{p}<0,001$ & 0,968 \\
Sul & 0,906 & $-1801,427$ & $\mathrm{p}<0,001$ & 0,936 \\
Centro-oeste & 0,570 & $-1131,261$ & $\mathrm{p}<0,001$ & 0,794 \\
Brasil & 0,588 & $-1166,111$ & $\mathrm{p}<0,001$ & 0,974 \\
\hline
\end{tabular}

Fonte: SINAN, Ministério da Saúde.

A análise da série temporal dos coeficientes de incidência de Aids no grupo etário de 50 anos e mais para o Brasil, tomando por base a estimativa de incidência esperada a partir da incidência observada até 1997 , revela que após um aumento em torno de $10 \%$ no ano de 1998, houve redução progressiva da diferença entre a incidência notificada e a estimada, até atingir o ano 2001. Após este ano, a incidência observada volta a ser superior que a esperada progressivamente, até alcançar um aumento de 7,22\% em 2003 (Gráfico 1).

Fazendo a mesma análise para as macrorregiões, vemos que a Região Norte (Gráfico 2), embora tenha apresentado oscilações na diferença entre as incidências observadas e esperadas, mostrou-se menor a partir de 1998 até 2001 (-45\%), sendo este ano o ponto de maior divergência entre as incidências espera- 
Gráfico 1 - Coeficiente de incidência (100.000 habitantes) observado e esperado de Aids no grupo etário de 50 anos e mais, segundo ano de diagnóstico, Brasil, 1990-2003

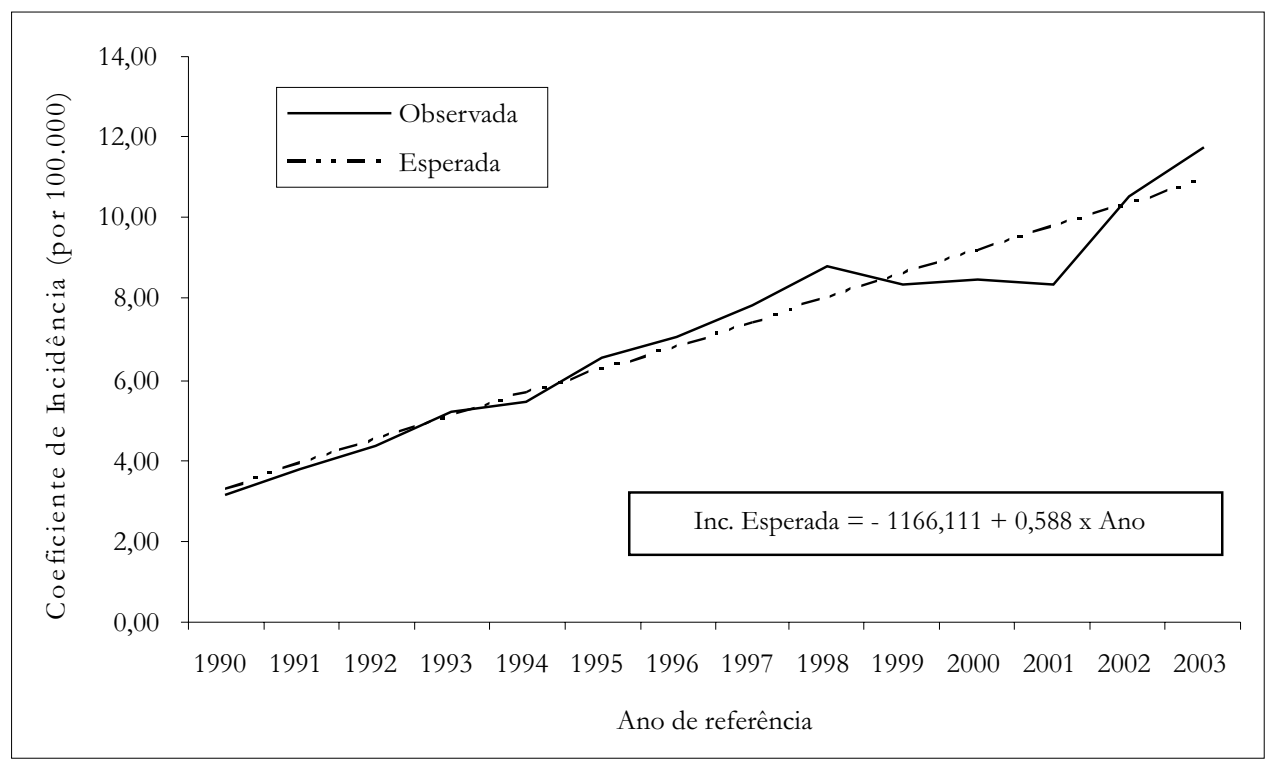

Gráfico 2 - Coeficiente de incidência (100.000 habitantes) observado e esperado de Aids no grupo etário de 50 anos e mais, segundo ano de diagnóstico, Norte, 1990-2003

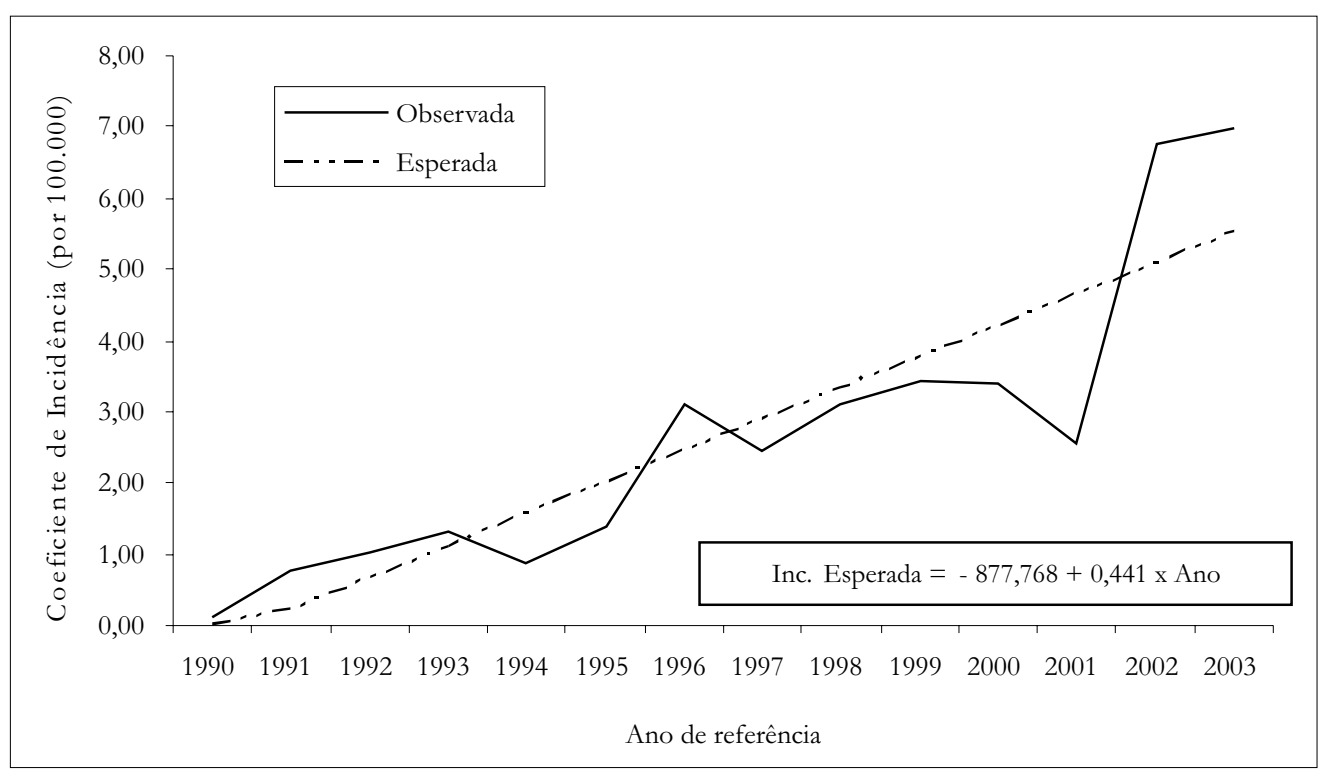


das e observadas em todas as regiões analisadas, sofrendo um brusco incremento positivo na diferença entre as incidências em 2002 $(32,87 \%)$ e posterior desaceleração desse aumento em 2003 (26,27\%).

A Região Nordeste também apresenta oscilações na tendência em todo o período estudado, embora com taxas menos divergentes entre as incidências observadas e esperadas que a Região Norte, terminando em 2003 com quase $2 \%$ a menos que o coeficiente de incidência estimado (Gráfico 3). O Sudeste brasileiro, após variações alternadas na tendência, mostra em 1998 um aumento de quase $11 \%$ em relação à

Gráfico 3 - Coeficiente de incidência (100.000 habitantes) observado e esperado de Aids no grupo etário de 50 anos e mais, segundo ano de diagnóstico, Nordeste, 1990-2003

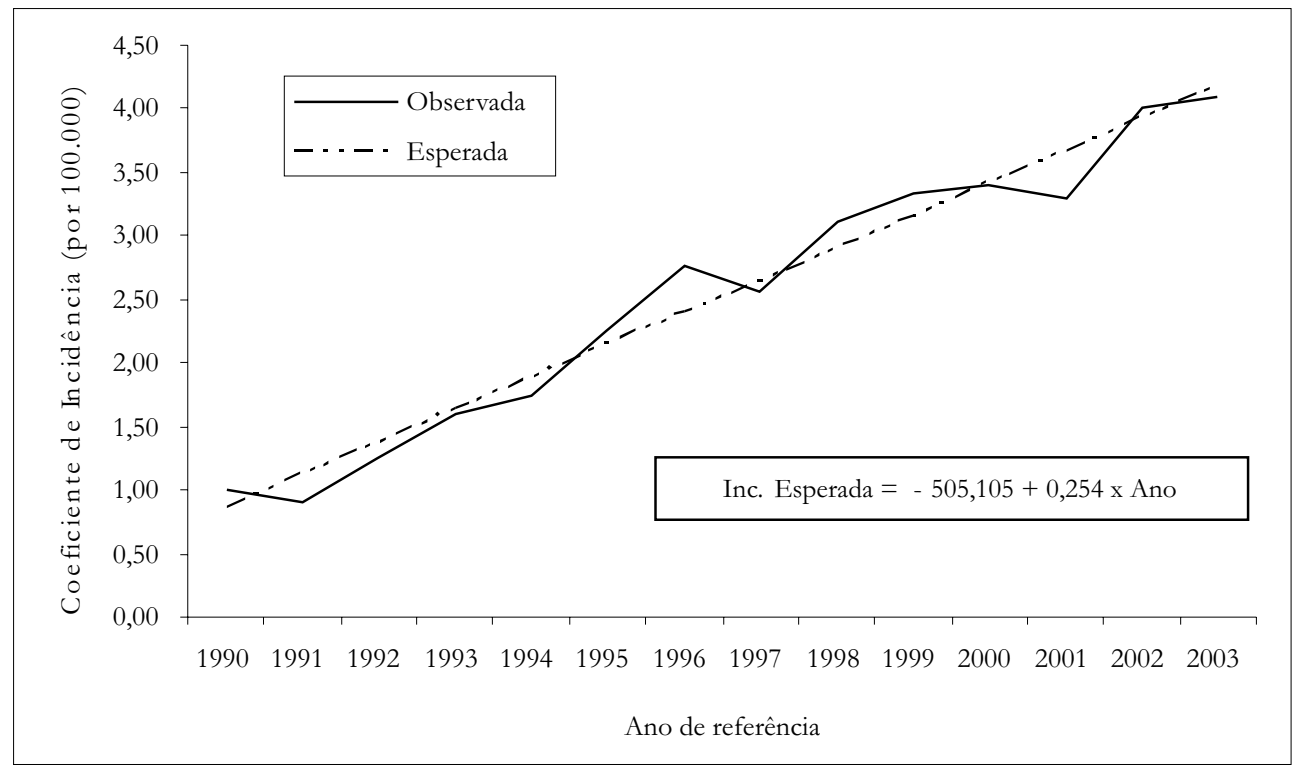

incidência esperada. Da mesma forma que o Brasil, a partir de 1998, o Sudeste apresenta um decremento progressivo dessa diferença até 2001 (-15\%), com desacelera- ção acentuada da queda em 2002 e posterior aumento das taxas observadas, atingindo quase $7 \%$ a mais que a incidência esperada em 2003 (Gráfico 4). 
Gráfico 4 - Coeficiente de incidência (100.000 habitantes) observado e esperado de Aids no grupo etário de 50 anos e mais, segundo ano de diagnóstico, Sudeste, 1990-2003

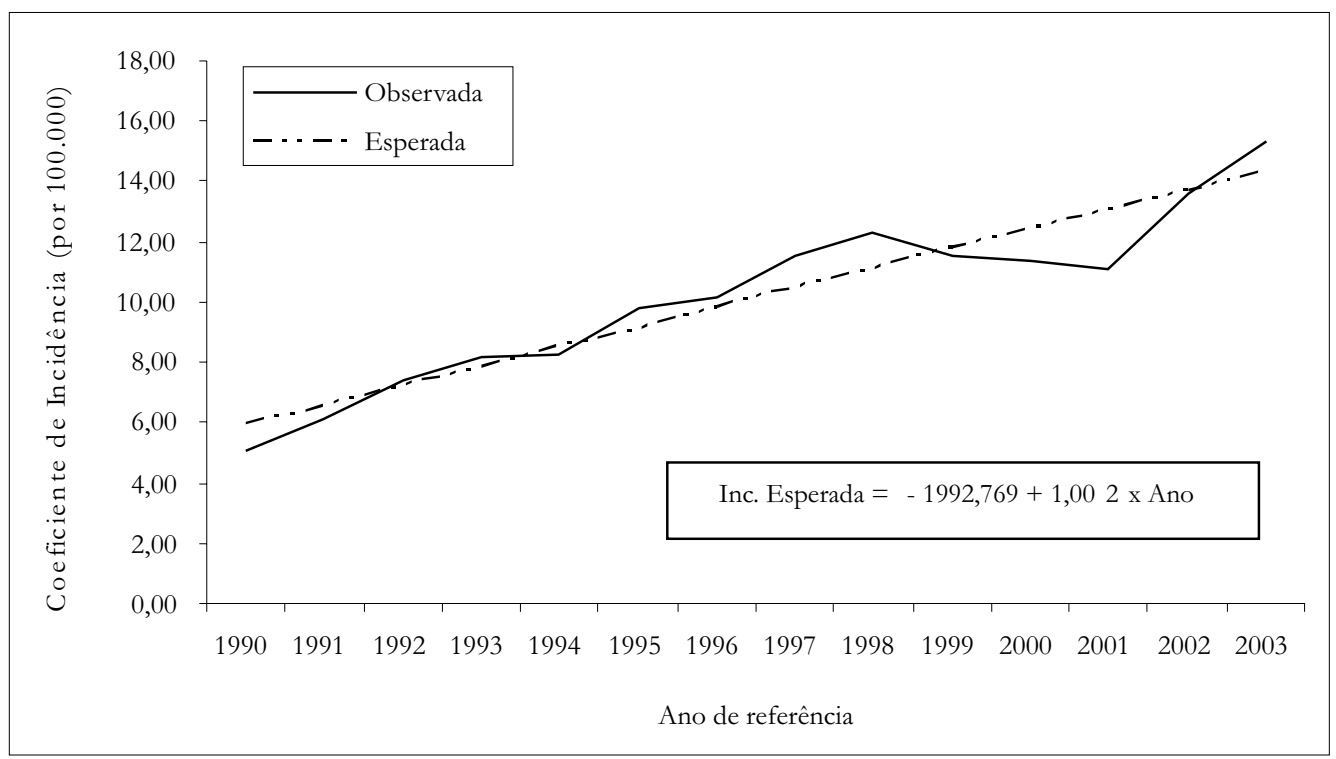

A Região Sul, semelhantemente ao que ocorreu com o Brasile Sudeste, apresenta aumento da incidência em relação ao esperado de quase $17 \%$ em 1998, com posterior redução progressiva até 2001 (-10,5\%), voltando a mostrar incremento positivo a partir desse ano, alcançando quase $5 \%$ a mais que o esperado em 2003 (Gráfico 5). A semelhança entre as tendências do Brasil com as regiões Sudeste e Sul deflagra a importância da influência dessas macrorregiões na epidemia do país.
No Centro-Oeste, a tendência de Aids no grupo estudado se aproxima mais do padrão da Região Norte, onde a incidência observada esteve sempre abaixo da esperada no período entre 1998 e 2001, tendo o momento de maior aceleração de queda no ano 2000 $(-23,7 \%)$, havendo, após 2002, mudança no comportamento da epidemia até atingir quase $25 \%$ a mais que a incidência esperada em 2003 (Gráfico 6). 
Gráfico 5 - Coeficiente de incidência (100.000 habitantes) observado e esperado de Aids no grupo etário de 50 anos e mais, segundo ano de diagnóstico, Sul, 1990-2003

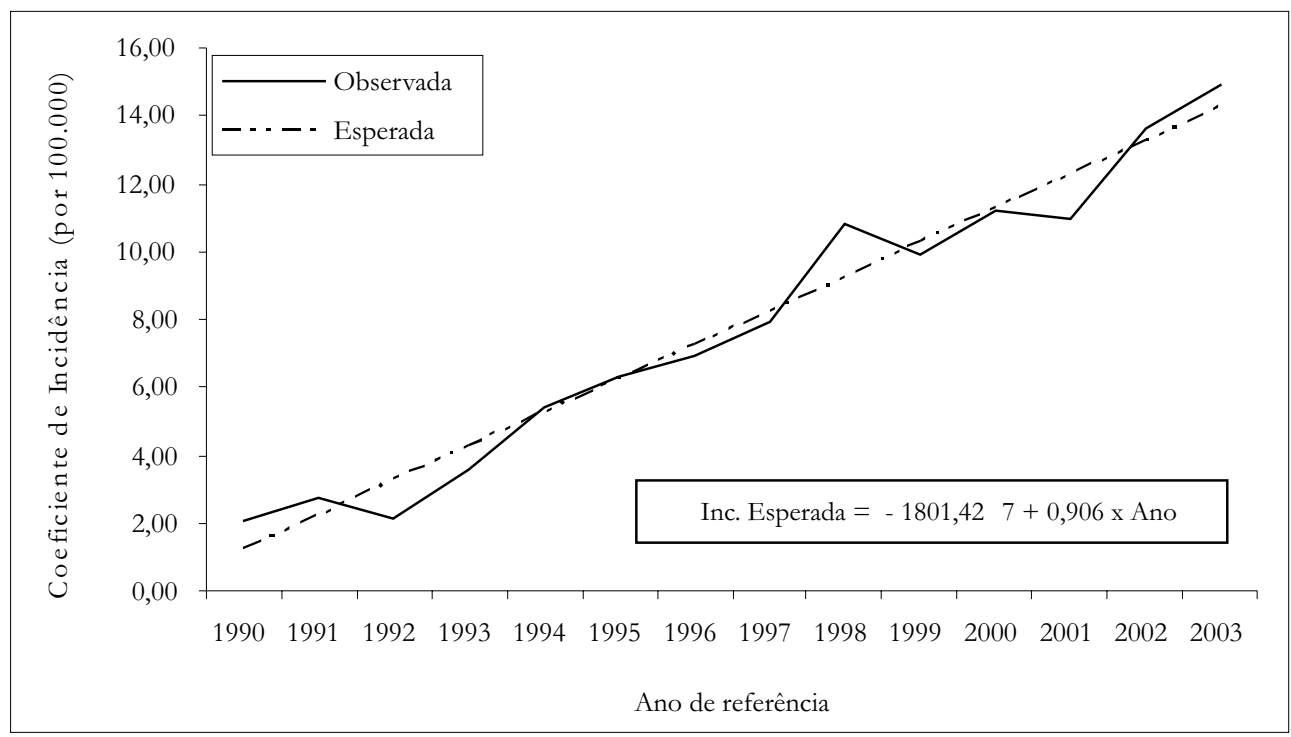

Gráfico 6 - Coeficiente de incidência (100.000 habitantes) observado e esperado de Aids no grupo etário de 50 anos e mais, segundo ano de diagnóstico, Centro-Oeste, 1990-2003

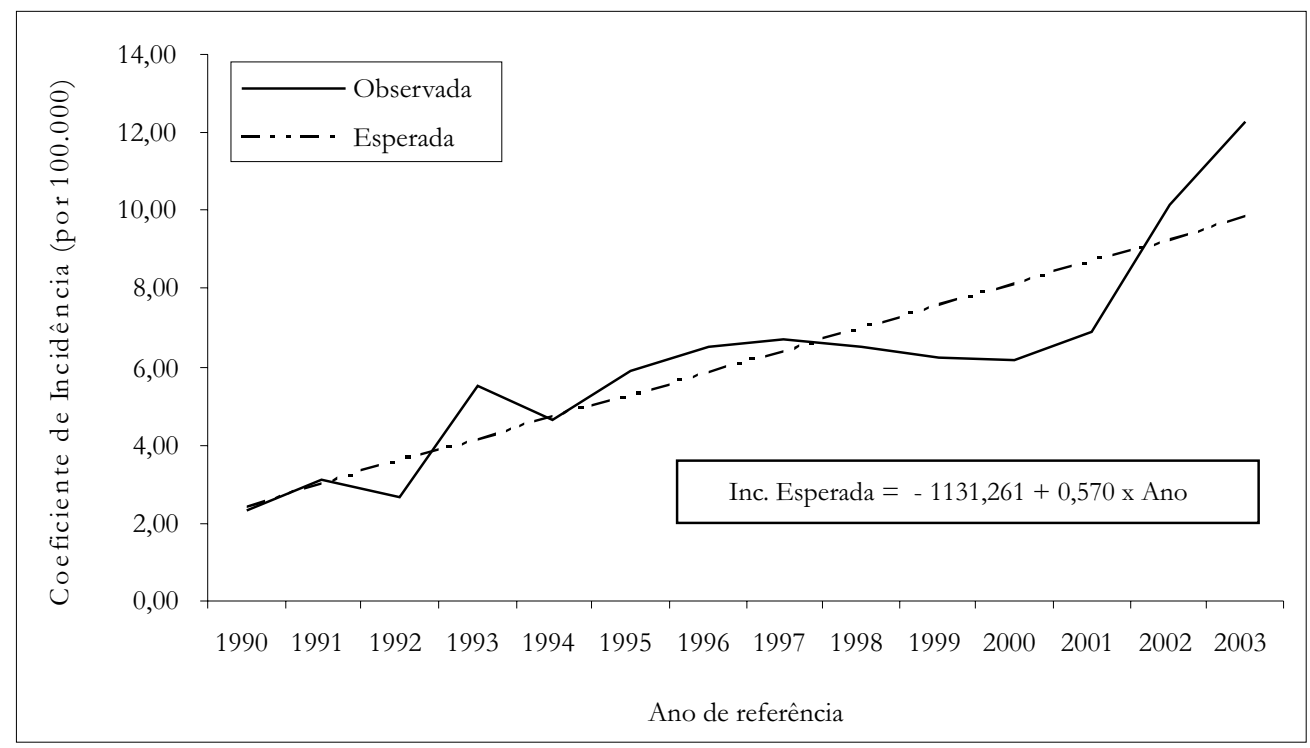


A comparação das taxas de incidência observadas e esperadas acumuladas no período de 1998 a 2003 revelaram tendências esperadas maiores do que as observadas em todas as regiões, exceto o Nordeste.

Analisando os anos extremos da série, observamos um altíssimo percentual de crescimento da incidência da Aids em pessoas de 50 anos e mais. No Brasil, as taxas variaram de 3,13, em 1990, para 11,73 casos por 100.000 habitantes em 2003, mostrando aumento de $375 \%$. Este foi seguido por todas as macroregiões: $6.336 \%$ no Norte, $720 \%$ no Sul, $522 \%$ no Centro-Oeste, $405 \%$ no Nordeste, e 300\% na Região Sudeste (Gráficos 1, $2,3,4,5$ e 6$)$.

Em relação aos anos extremos do período posterior à introdução dos medicamentos para disfunção erétil (1998 e 2003), percebemos que as taxas de incidência em todas as regiões continuam em tendência de crescimento, embora em arrefecimento: $133 \%$ no Brasil e $223 \%$ na Região Norte; $189 \%$ no Centro-Oeste; $139 \%$ no Sul; $132 \%$ no Nordeste e $125 \%$ para a macrorregião Sudeste (Gráfi$\cos 1,2,3,4,5$ e 6$)$.

\section{DISCUSSÃO}

Da mesma forma que a epidemia como um todo, na faixa etária de 50 anos e mais, a Aids prevalece nas macrorregiões mais desenvolvidas do país. O Sudeste e o Sul sempre alcançaram a grande maioria dos casos em todos os anos da série, confirmando os estu- dos anteriores. ${ }^{4,712}$ A baixa participação das outras regiões, principalmente a Norte, pode ser reflexo de uma introdução retardada da epidemia de Aids nessas macrorregiões e de problemas com o sistema de vigilância epidemiológica. ${ }^{5,9}$

O Brasil e todas as grandes regiões apresentaram tendência de crescimento das taxas no período entre 1990 e 1997, estatisticamente significante, embora de forma não-homogênea em todo território. As regiões Sudeste e Sul apresentaram maiores taxas de variação média anual para o referido período, bem acima das encontradas para o Brasil e as outras regiões, o que refuta a literatura, quando esta retrata a epidemia em todas as faixas etárias, que colocam sempre as maiores taxas de crescimento para as regiões Norte, Nordeste e Centro-Oeste. ${ }^{5,12} \mathrm{O}$ fato de a epidemia de Aids em pessoas com 50 anos e mais prevalecer nos grandes centros desenvolvidos assemelha-se ao início da epidemia em todas as faixas etárias, quando sua incidência era quase exclusiva nessas regiões. ${ }^{5,9,12}$ Vale ressaltar que a taxa de variação média anual da Região Norte pode estar somente "aparentemente" elevada, influenciada pela maior flutuação dos dados, uma vez que apresenta reduzida incidência, principalmente nos primeiros anos da série analisada.

As tendências mais recentes de estabilização da incidência da Aids em todas as faixas etárias no Brasil e nas regiões Sudeste e Sul, segundo resultados de pesquisas anteriores, ${ }^{4,8,14}$ não foram confirmadas quando comparamos com a evolução da doença no grupo etário de 50 anos e mais. 
A análise dos dados revela que houve aumento expressivo da incidência de Aids em pessoas com 50 anos e mais em todas as regiões estudadas, quando comparamos 1990 com 2003, com crescimento nas regiões variando de 300 (Sudeste) a 6.336\% (Norte), o que comprova a crescente participação dessa faixa etária na epidemia, indo contra tendências de estabilização ou redução da incidência de Aids em outros componentes etários de nossa população. $5,8,9$

Em relação ao período posterior à introdução dos medicamentos para disfunção erétil, a incidência continua com tendência de crescimento, embora com menor velocidade, mostrando crescimento entre 125 e $223 \%$, quando comparamos 1998 com 2003.

Embora apresente tendência crescente das taxas de incidência de Aids em pessoas com 50 anos e mais em toda série, tanto o Brasil como todas as macrorregiões mostraram incidências observadas menores que as esperadas para o período entre 1998 e 2003, com exceção da Região Nordeste. Tal resultado é semelhante ao encontrado em outro estudo, quando utilizou metodologia similar para avaliar a incidência em todas as faixas etárias, também no período de 1990 a $2003 .{ }^{8}$ Isso demonstra que tanto para todas as faixas etárias quanto para pessoas com 50 anos e mais, a epidemia de Aids apresenta velocidades maiores de crescimento para o período de 1990 a 1997.

Componentes importantes para explicar as diferenças de tendências da epidemia segundo as macrorregiões são: a evolução da epidemia, o grau de implementação das medidas de prevenção, o conhecimento da população sobre a Aids, o nível de escolaridade e outros fatores socioeconômicos. ${ }^{8,9}$

A regressão linear se apresentou como um bom modelo explicativo para a epidemia de Aids na faixa etária de 50 anos e mais, na série histórica de 1990 a 2003, já que, em todas as regiões estudas, o Coeficiente de Determinação $\left(\mathrm{R}^{2}\right)$ se mostrou próximo de 1, o que deflagra a ótima linearidade do modelo.

Hipóteses surgem para explicar o fenômeno do aumento da incidência da Aids na faixa etário de 50 anos e mais, principalmente quando analisamos os anos extremos da série aqui estudada $(1990$ e 2003). Fatores como o aumento da expectativa de vida, a melhora da qualidade de vida de pessoas com 50 anos e mais, aumento das relações sexuais, disposição de tecnologia que melhore e prolongue a performance sexual dos idosos, menor uso de preservativos nessa faixa etária, não-inclusão e não-identificação com campanhas preventivas de DST/Aids, redução da função imune, mudanças biológicas, dentre outros fatores, podem vir a ser comprovados como influência decisiva do aumento da epidemia, através de estudos posteriores e específicos que contemplem aspectos socioeconômicos, demográficos e comportamentais dessa faixa etária. ${ }^{2,4,6,9,10,11,13}$

Os resultados aqui apresentados apontam para uma provável influência retardada dos vários fatores listados acima, dentre eles os medicamentos para disfunção 
erétil, como uma possível explicação para descrever a tendência de Aids no grupo etário de 50 anos e mais. Retardada porque, infelizmente, a não-disposição de dados de infecção pelo HIV em adultos maduros e idosos - a infecção não é objeto de notificação compulsória no Brasil, salvo os casos em gestantes e crianças expostas ao risco de transmissão vertical - dificulta a análise precisa da magnitude desses fatores na epidemia de Aids, já que é bem claro na literatura que a enfermidade Aids pode apresentar períodos de latência longos. Assim, a própria infecção pelo HIV seria mais sensível para a influência imediata de tais fatores. Contudo, estudos que trabalham com a infecção pelo HIV se fazem a partir de estimativas, o que não é objetivo desta pesquisa, pois gostaríamos de trabalhar com dados reais de notificação da epidemia do país.

Dessa forma, os resultados aqui mostrados devem ser tomados com certa cautela, pois os seis anos analisados para o período posterior à introdução dos medicamentos para disfunção erétil no país, provavelmente são insuficientes para uma descrição mais fiel da sua possível influência na epidemia de Aids em pessoas com 50 anos e mais, embora a literatura aponte esses medicamentos como uma possível variável relacionada ao aumento de Aids nessa faixa etária, necessitando de estudos futuros que investiguem tal associação. ${ }^{2,9}$

Em relação às limitações metodológicas encontradas na realização do estudo, a primeira é a confiabilidade quali-quantitativa da informação prestada, por se tratar de estudo com dados secundários, onde não há controle do pesquisador em relação a erros decorrentes de digitação e registro e à própria cobertura da informação. Apesar destas ressalvas, foram considerados válidos, por se tratar dos dados oficiais do Programa Nacional de DST e Aids.

Outra limitação se refere aos pequenos números de casos de Aids notificados em idosos (60 anos e mais) para algumas macrorregiões do país, principalmente nos primeiros anos da série estudada, o que pode levar a dificuldades de análise, devido às possíveis flutuações dos dados. A alternativa utilizada para minimizar o problema foi ampliar a faixa etária estudada para pessoas com idade igual ou superior a 50 anos, seguindo a mesma condução metodológica de outros estudos. ${ }^{1,9}$

\section{CONCLUSÕES}

A partir da análise dos resultados deste estudo, conclui-se que:

1 - Oitenta e quatro por cento dos casos de Aids em pessoas com 50 anos e mais, no período de 1990 a 2003, concentram-se nas regiões Sudeste e Sul, o que demonstra serem essas regiões as de maior magnitude para a epidemia de Aids no país.

2 - O Brasil apresentou uma incidência de Aids na faixa etária de 50 nos e mais no total da série estudada de 7,45 casos por 100.000 habitantes. Em relação às macrorregiões, a incidência total também foi maior nas regiões 
Sudeste (10,5/100.000) e Sul (8,3/100.000), seguidas pelas regiões Centro-Oeste $(7,0 /$ 100.000), Norte $(3,0 / 100.000)$ e Nordeste $(2,65 / 100.000)$.

3 - As taxas de variação média anual para o Brasil $(0,588)$ e todas as macrorregiões, no período entre 1990 e 1997, mostraram tendência crescente e estatisticamente significante da incidência de Aids no grupo etário de 50 anos e mais, sendo maiores no Sudeste $(1,002)$ e Sul $(0,906)$, seguidas pelo Centro-Oeste $(0,570)$, Norte $(0,441)$ e Nordeste $(0,254)$.

4 - Quando comparamos anos extremos da série estudada (1990 e 2003), todas as regiões analisadas apresentaram extraordinário crescimento das taxas de incidência de Aids em pessoas com 50 anos e mais, sendo o aumento de $375 \%$ para o Brasil, $6.336 \%$ para a Região Norte, $720 \%$ para o Sul, $522 \%$ para o Centro-Oeste, $405 \%$ para o Nordeste e $300 \%$ para a macrorregião Sudeste.

5 - Em relação ao período posterior à introdução dos medicamentos para disfunção erétil, todas as regiões mantiveram tendência crescente das taxas de incidência, embora com menor velocidade: $133 \%$ no Brasil, $223 \%$ na Região Norte; $189 \%$ no Centro-Oeste; $139 \%$ no Sul; $132 \%$ no Nordeste e $125 \%$ para o Sudeste, quando comparamos 1998 com 2003.

6 - Comparando-se as incidências observadas e esperadas para o período posterior à introdução dos medicamentos para disfunção erétil (1998 a 2003), as taxas observadas foram menores que as estimadas em todas as regiões estudadas, salvo na macrorregião Nordeste;

7 - A tendência de Aids na faixa etária de 50 anos e mais apresentou maiores taxas de crescimento no período entre 1990 e 1997, mantendo esse crescimento no período posterior à introdução de medicamentos para disfunção erétil, só que em menor velocidade.

\section{NOTAS}

a Mestrado em Saúde Coletiva, Programa Integrado em Saúde Coletiva (PIPASC), Universidade Federal de Pernambuco. Recife, PE, Brasil. E-mail: jailsonsaudecoletiva@yahoo.com.br

b Doutorado em Saúde Coletiva, Departamento de Medicina Social, Programa Integrado em Saúde Coletiva (PIPASC) - Universidade Federal de Pernambuco, Recife, PE, Brasil.

E-mail: mdpaz.s@oi.com.br

c Mestrado em Saúde Coletiva Centro de Pesquisa Ageu Magalhães/Fiocruz. Recife, PE. Brasil.

E-mail: ulisses@cpqam.fiocruz.br

Fomento: CAPES (bolsa de mestrado)

\section{REFERÊNCIAS BIBLIOGRÁFICAS}

1. Alves FAP. Aids e envelhecimento: características dos casos com idade igual ou maior a 50 anos em Pernambuco, 1990 a 2000. [dissertação]. Recife: Universidade Federal de Pernambuco, Programa Integrado de Pósgraduação em Saúde Coletiva; 2002. 80p.

2. Berg OLD. Afinal, o que é disfunção erétil? Verdades e mentiras sobre a impotência sexual. Rio de Janeiro: Dunya; 2000. 155p.

3. Berquó E, et al. Comportamento sexual da população brasileira e percepções do HIV/ aids: relatório final. Brasília: Ministério da Saúde;1999. Mímeo. 
4. Brito AM, Castilho EA, Szwarcwald CL. Aids e infecção pelo HIV no Brasil: uma epidemia multifacetada. Rev Soc Bras Med Trop 2000 abr./jun; 34: 207-17.

5. Chaimowicz F. Os idosos brasileiros no século XXI: demografia, saúde e sociedade. Belo Horizonte: Post Graduate do Brasil;1998. 128p.

6. Dhalia C, Barreira D, Castilho EA. A aids no Brasil: situação atual e tendências. Bol Epidemiol AIDS 2000; 13(1): 3-13.

7. Dourado I, et al. Tendência da epidemia de Aids no Brasil após a terapia anti-retroviral. Rev Saúde Pública 2006; 40 (Supl): 9-17.

8. Feitoza AR, Sousa AR, Araújo MFM. A magnitude da infecção pelo HIV-Aids em maiores de 50 anos no município de fortaleza-CE. Jornal brasileiro de doenças sexualmente transmissiìveis : DST 2004; 16( 4): 32-7.

9. Kalache A, Keller I. The greying world: a challenge for the 21 st century. Sci Prog 2000; 83(1): 33-54.
10. Linsk NL. HIV older adults: age-specific issues in prevention and treatment. AIDS $\operatorname{Read} 2000 ; 10(7)$ : 430-40.

11. Lopes CR. Os novos números da epidemia. Radis : comunicaçaPo em sauide. 2006 jan.; 41: 9-11.

12. Matsushita RY, Santana RS. Uma análise da incidência dos casos de aids por faixa etária. Bol Epidemiol AIDS 2001 abr./jun; 14(2): 3-5.

13. Ministério da Saúde (Br). Análise do banco de dados nacional de Aids, 1980 a 2006 e gestante HIV+, 2000 a 2006. Bol Epidemiol AIDS 2006 jan./jun; 3(1) 7-45.

14. Szwarcwald CL, et al. a disseminação da epidemia de Aids no Brasil, no período de 1987-1996: uma análise espacial. Cad Saúde Pública 2000; 16 (Supl 1):7-19.

Recebido em: 28/3/2007

Aceito: 21/5/2007 